\section{Arktische Allergien}

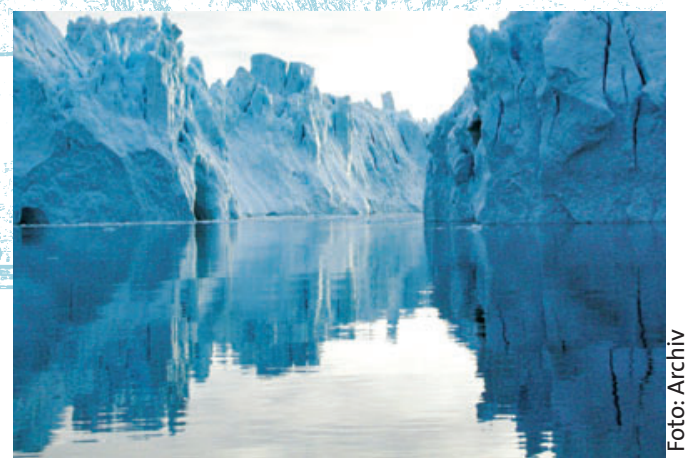

$\mathrm{n}$ den meisten Industrieländern ist die Epidemiologie atopischer Erkrankungen gut dokumentiert. Wie sieht es jedoch in Grönland aus? Dänische Wissenschaftler untersuchten die spezifischen IgE-Werte bezüglich acht häufiger inhalativer Allergene in den Blutproben von rund 850 Grönländern (400 Proben aus dem Jahr 1987 und ca. 450 aus dem Jahr 1998). Dabei konnten sie im Vergleich der beiden Jahre annähernd eine Verdoppelung der AtopiePrävalenz von $10 \%$ auf $19 \%$ protokollieren. Besonders dramatisch war der Anstieg in der Gruppe der 15- bis 19-Jährigen, bei denen im Jahr 1998 Atopien fast 5-mal so häufig waren wie $1987 . \quad n z$

Krause TG et al. Lancet 2002; 360: 691-2

\title{
Alter schützt vor Allergien nicht
}

W ächst sich eine Allergie mit den Jahren aus? Etliche Querschnittstudien scheinen diese populäre These zu stützen, indem sie nachweisen, dass die Prävalenz allergischer Erkrankungen mit dem Alter stetig abnimmt. Englische Wissenschaftler aus Nottingham jedoch konnten jetzt das Gegenteil belegen: In einer Longitudinalstudie, in der sie an denselben 1.339 Probanden Prick-Tests vornahmen - einmal 1991 und einmal 2000 -, fanden sie heraus,

\section{Nickelschleuder Euro}

$M$ etallurgen von der Eidgenössischen Technischen Hochschule in Zürich haben die neuen Euro-Münzen genau unter die Lupe genommen und Peinliches zu Tage gefördert. Die 1und 2-Euro-Münzen überschreiten die von der EU 1994 festgelegten Grenzwerte zur Nickelfreisetzung von Schmuck um das 240- bis 320fache. Grund dafür ist nicht nur der hohe Nickelgehalt, sondern vor allem der bimetallische Aufbau der Mün-

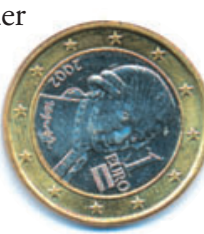

dass die allergische Disposition der einzelnen Testpersonen nach neun Jahren quasi unverändert war. Die geringere Prävalenz im Alter ist also nicht auf eine nachlassende Sensiblisierung des Individuums zurückzuführen, sondern ausschließlich auf die allgemeine Zunahme von Allergien in den nachfolgenden Generationen.

Broadfield E et al. J Allergy Clin Immunol 2002; 109: 969-74

\section{Allergene wirken schon in utero}

A uch das fetale Immunsystem reagiert schon auf seine Umwelt: Eine Gruppe von deutschen Wissenschaftlern fand bei rund einem Drittel von insgesamt 1.332 untersuchten Neugeborenen aus München oder Leipzig IgE-Antikörper im Nabelschnurblut. Durch die Passage geringer Mengen von Antigenen durch die Plazentaschranke produzierten die Feten bereits im Mutterleib Immunglobuline. Die IgEAntikörper gegen Katzenallergene standen dabei in einer direk- ten Beziehung zu der Menge an Allergenen, die die Mediziner aus dem Staub der mütterlichen Matratzen isoliert hatten. Bei den Milbenallergenen nahm die IgE-Konzentration mit ansteigender Allergenmenge zunächst zu und fiel dann jedoch wieder ab. Ein

Kuriosum am Rande: Sowohl die Menge an Katzen- wie auch an Milbenallergenen war in Leipzigs Matratzen doppelt so hoch wie in München.

$n z$

\section{Heinrich J et al.}

Eur Respir J 2002; 20: 617-23

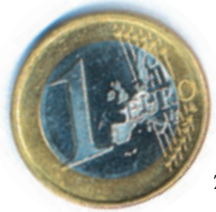

zen: Die Kontaktstelle zwischen Ring und innerem Metall (vgl. Titelbild) bildet ein galvanisches Element, bei Kontakt mit Handschweiß fließt Strom. Dadurch korrodiert die Münze und allergene Nickelionen werden freigesetzt. Nickel wird den Münzen vor allem wegen seiner magnetischen Eigenschaften beigemischt, die ein Erkennen der Münzen in Automaten erleichtern. es

Nestle FO et al. Nature 2002; 419: 132

\section{Laser gegen Asthma?}

sterreichische Pädiater haben ei-
nen eleganten Versuch durchgeführt, um die Wirksamkeit einer Laserakupunktur gegen Asthmaanfälle bei Kindern zu prüfen. Die kleinen Probanden mussten vier Minuten lang Luft mit einer Temperatur von $-10^{\circ} \mathrm{C}$ hyperventilieren, wodurch eine reproduzierbare Bronchokonstriktion induziert werden sollte. In randomisierter Reihenfolge wurden die Kinder außerdem entweder mit Laser- oder Scheinlaserakupunktur vorbehandelt. Die Messung der Lungenfunktion zeigte die zu erwartenden Einschränkungen, jedoch keinen Effekt der Laserakupunktur.

Gruber W et al. Thorax 57; 2002: 222-5 